\title{
Closed 3-braids are nearly fibred
}

\author{
YI NI \\ Department of Mathematics, Princeton University, Princeton, New Jersey 08544 \\ Email: yni@math.princeton.edu
}

\begin{abstract}
Ozsváth and Szabó conjectured that knot Floer homology detects fibred links. We will verify this conjecture for closed 3-braids, by classifying fibred closed 3-braids. In particular, given a nontrivial closed 3-braid, either it is fibred, or it differs from a fibred link by a half twist. The proof uses Gabai's method of disk decomposition.
\end{abstract}

AMS Classification 57M27; 57R58.

Keywords knot Floer homology, 3-braids, fibred links, disk decomposition.

\section{Introduction}

Knot Floer homology was introduced by Ozsváth and Szabó [7], and independently by Rasmussen [11]. The Euler characteristic of knot Floer homology gives rise to the Alexander-Conway polynomial. Knot Floer homology contains a lot of information about the knot or link. For example, it detects the genera of classical links. Namely, we have the following theorem due to Ozsváth and Szabó ([9], see also [5]).

Theorem Suppose $L$ is an oriented link in $S^{3}$. Let $\chi(L)$ be the maximal Euler characteristic of the Seifert surfaces bounded by $L$, and $\mathfrak{i}(L)=\frac{|L|-\chi(L)}{2}$, where $|L|$ is the number of components of $L$. Then

$$
\mathfrak{i}(L)=\max \{i \mid \widehat{H F K}(L, i) \neq 0\} .
$$

We always refer $\widehat{H F K}(L, \mathfrak{i}(L))$ as the topmost term in the knot Floer homology. We say an oriented link $L$ is fibred, if the complement of $L$ fibers over the circle, and $L$ is the boundary of the fiber. We say the knot Floer homology of a link is monic, if the topmost term is isomorphic to $\mathbb{Z}$. Ozsváth and Szabó proved that if the link is fibred, then the knot Floer homology is monic ([8], see also [5]). Thus we naturally have the following conjecture (see [10]):

Conjecture 1.1 If a link in $S^{3}$ has monic knot Floer homology, then it is a fibred link. 
Not many interesting cases were tested for this conjecture. In the knot table, there are exactly thirteen 12-crossing non-fibred knots, each of which has monic Alexander polynomial, and degree of the Alexander polynomial precisely gives the genus [2]. According to some unpublished computations done by Rasmussen, Ni and Juhász, these knots do not have monic knot Floer homology. Moreover, the results in [6] give positive theoretical evidence to the conjecture.

In this paper, we will verify the conjecture for closed 3-braids. Our geometric result is

Theorem 1.2 Suppose link $L \in S^{3}$ is the closure of a 3-braid, then exactly one of the following 3 cases happens:

i) $L$ is the 3-component trivial link;

ii) $L$ is fibred;

iii) $L$ or its mirror image is the closure of a nondecreasing positive word $P$. Moreover, either $P$ is a power of one of $a_{1}, a_{2}, a_{3}$, or $P$ is started with $a_{1}$ and ended with $a_{3}$. Hence in the corresponding braid diagram, after adding a half twist, we get a fibred link.

The exact meaning of case iii) will become clear after Definition 2.2. Our theorem, together with some simple computations of knot Floer homology, gives the following

Corollary 1.3 A closed 3-braid is fibred if and only if it has monic knot Floer homology.

Remark 1.4 Closed 3-braids were classified by Birman and Menasco as links [1]. It is proved there that a generic closed 3-braid is represented by a unique conjugacy class of 3 -braids.

Remark 1.5 We are informed by Alexander Stoimenow that the classification of fibred closed 3-braids has been obtained in [13], with the assistance of Hirasawa and Murasugi.

The paper is organized as follows: In Section 2, we will compute the topmost terms in the knot Floer homology of closed 3-braids. The computation uses a result of Xu [14]. In Section 3, we apply Gabai's method of disk decomposition to prove Theorem 1.2.

Acknowledgements. We wish to thank David Gabai, András Juhász and Zoltán Szabó for some helpful conversations. We also wish to thank Joan Birman for some comments on the paper. We are especially grateful to Jacob 
Rasmussen, who pointed out a crucial mistake in an earlier version of this paper, and to Xingru Zhang, from whose lecture the author learned Xu's work on 3-braids.

The author is partially supported by the Centennial fellowship of the Graduate School at Princeton University.

\section{Knot Floer homology of closed 3-braids}

In this section, we will compute the topmost terms in the knot Floer homology of closed 3-braids. Although the result can be deduced from our main theorem, the computation here has its own interest. And the computation motivates our main theorem.

Of course, the computation becomes easier, if we already know what the genus of a 3-braid is. Fortunately, this problem was solved by $\mathrm{Xu}$ [14]. In order to explain her result, we need some preparation.

Notation 2.1 $B_{3}$ denotes the group of 3-braids, $\sigma_{1}$ and $\sigma_{2}$ are the standard generators of $B_{3}$. Instead of the standard presentation, we use three generators $a_{1}=\sigma_{1}, a_{2}=\sigma_{2}, a_{3}=\sigma_{2} \sigma_{1} \sigma_{2}^{-1}$, and the presentation

$$
B_{3}=<a_{1}, a_{2}, a_{3} \mid a_{2} a_{1}=a_{3} a_{2}=a_{1} a_{3}>\text {. }
$$

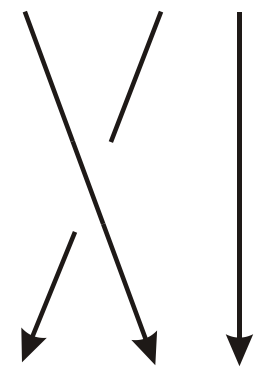

$a_{1}=\sigma_{1}$

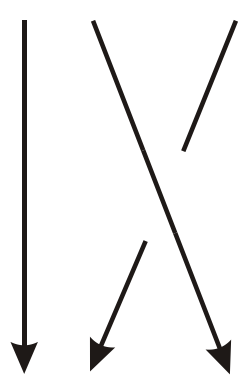

$a_{2}=\sigma_{2}$

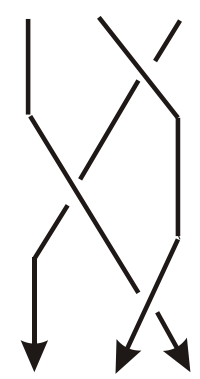

$a_{3}=\sigma_{2} \sigma_{1} \sigma_{2}^{-1}$

Figure 1 The generators of $B_{3}$.

The advantage of such presentation is, one can draw $a_{1}, a_{2}, a_{3}$ cyclically on a cylinder, thus we can permute the roles of $a_{1}, a_{2}, a_{3}$ cyclically. The reader is encouraged to figure this out by himself/herself.

If $w \in B_{3}$, then $\bar{w}$ denotes its inverse. Let $\alpha=a_{2} a_{1}=a_{3} a_{2}=a_{1} a_{3}$. The following relations will be useful to us:

$$
a_{i} \bar{a}_{j}=\bar{a}_{i+1} a_{j+1}, \quad \alpha \bar{a}_{i}=a_{i+1}, \quad \bar{a}_{i} \alpha=a_{i-1} .
$$


Definition 2.2 Suppose $P=a_{\varepsilon_{1}} \cdots a_{\varepsilon_{n}}$ is a positive word. We say $P$ is nondecreasing if for each $j \in\{1, \ldots, n-1\}, \varepsilon_{j+1}=\varepsilon_{j}$ or $\varepsilon_{j}+1$, where the subscript for $a$ is understood cyclically. $P$ is strictly increasing, if for each $j \in\{1, \ldots, n-1\}, \varepsilon_{j+1}=\varepsilon_{j}+1$.

Theorem 2.3 (P.J. Xu) Every conjugacy class in $B_{3}$ can be represented by a shortest word in $a_{1}, a_{2}, a_{3}$ which is unique up to symmetries. The word has one of the following forms:

i) $\alpha^{k} P$;

ii) $N \alpha^{-k}$;

iii) $N P$.

Here $k \geq 0, \bar{N}$ and $P$ are nondecreasing positive words, $P$ or $N$ may be empty.

Moreover, the minimal Seifert surface of the corresponding closed braid can be constructed from this word.

We briefly explain how to construct the Seifert surface from a word $w$. We first resolve the braid to a 3 -component trivial link, bounding 3 disjoint disks. Then for each letter in $w$, one attaches a twisted band to connect two of the 3 disks. This surface is called the Bennequin surface of the word $w$, denoted by $B_{w}$. It has Euler characteristic $3-l(w)$, where $l(w)$ is the length of $w$.

From now on, we also use the word $w$ to denote the corresponding 3-braid, if there is no confusion. Xu's theorem says that, for a shortest word $w$ as above, $\chi(w)=3-l(w)$.

Remark 2.4 If the subword $a_{i} a_{i}$ appears in $w$, one can replace it by a single $a_{i}$ to get a new word $w^{\prime}$. The Bennequin surface of $w$ is the plumbing of the Bennequin surface of $w^{\prime}$ with a Hopf band. It is easy to see the top terms in the knot Floer homology of $w$ and $w^{\prime}$ are isomorphic as abelian groups. Moreover, the closure of $w^{\prime}$ is fibred if and only if the closure of $w$ is fibred [3].

Given a reduced word $w$ in Xu's form, we can apply the previous "untwisting" operation repeatedly, until we get a word also in Xu's form, but now the $\bar{N}$ and/or $P$ are strictly increasing. We denote this new word by $U T(w)$.

Theorem 2.5 Suppose $L$ is the closure of a 3-braid $w . w$ is in the form in Theorem 2.3. We consider the word $U T(w)$. If $U T(w)$ is in the form of $N$ or $P$, and $l(U T(w))=3 t+1$ or $3 t+3,(t \geq 0)$, then

$$
\widehat{H F K}(L, \mathfrak{i}(L)) \cong \mathbb{Z} \oplus \mathbb{Z} .
$$

In other cases, $\widehat{H F K}(L)$ is monic, except when $L$ is the 3-component trivial link. 
We divide the theorem into several propositions.

Proposition 2.6 Suppose $w=\alpha^{k} P$ is a word in $X u$ 's form, $k>0$. L is the closure of $w$, then $L$ is fibred with fiber $B_{w}$. Here $B_{w}$ is the Bennequin surface of $w$.

Proof Suppose the first letter in $P$ is $a_{1}$, then $P=a_{1} P^{\prime} . w=\alpha^{k} a_{1} P^{\prime}=$ $\alpha^{k-1} a_{2} a_{1} a_{1} P^{\prime}$. Hence $B_{w}$ is the plumbing of a Hopf band with the Bennequin surface of $\alpha^{k-1} a_{2} a_{1} P^{\prime}=\alpha^{k} P^{\prime}$. By [3] we can reduce our problem to $\alpha^{k} P^{\prime}$, hence to $\alpha^{k}$ by induction. Our conclusion holds since $\alpha^{k}$ is a torus link.

Lemma 2.7 $L$ is in the form $N P, 1=l(N) \leq l(P)$, then $\widehat{H F K}(L)$ is monic.

Proof We can assume $N=\bar{a}_{2}$. We will prove our result by induction on $l(P)$. When $l(P)=1, N P=\bar{a}_{2} a_{1}$ or $\bar{a}_{2} a_{3}$, hence $L$ is the unknot. Now assume $l(P)>1$, and $P$ is strictly increasing.

If the last letter in $P$ is $a_{3}$, then $P$ can be written as $P^{\prime} a_{1} a_{2} a_{3}$. We have the skein relation for

$$
L_{-}=\bar{a}_{2} P^{\prime} a_{1} a_{2} a_{3}, \quad L_{0}=\bar{a}_{2} P^{\prime} a_{1} a_{3}, \quad L_{+}=\bar{a}_{2} P^{\prime} a_{1} \bar{a}_{2} a_{3} .
$$

And we have $\bar{a}_{2} P^{\prime} a_{1} a_{3} \sim \alpha \bar{a}_{2} P^{\prime}=a_{3} P^{\prime}$, (" $\sim$ " denotes conjugacy relation in $B_{3}$, ) hence $\chi\left(L_{0}\right) \geq \chi\left(L_{-}\right)+3$. In the local picture of the skein relation, if the two strands in $L_{-}$belong to the same component, then $\left|L_{0}\right|=$ $\left|L_{-}\right|+1$, and $\mathfrak{i}\left(L_{0}\right)<\mathfrak{i}\left(L_{-}\right)$; if the two strands in $L_{-}$belong to different components, then $\left|L_{0}\right|=\left|L_{-}\right|-1$, and $\mathfrak{i}\left(L_{0}\right)+1<\mathfrak{i}\left(L_{-}\right)$. In any case, using the surgery exact triangle [7], we get an isomorphism between $\widehat{H F K}\left(L_{-}, \mathfrak{i}\left(L_{-}\right)\right)$ and $\widehat{H F K}\left(L_{+}, \mathfrak{i}\left(L_{+}\right)\right)$.

As for $L_{+}$, we have $\bar{a}_{2} P^{\prime} a_{1} \bar{a}_{2} a_{3}=\bar{a}_{2} P^{\prime} a_{1} a_{1} \bar{a}_{2} \sim \bar{a}_{2}^{2} P^{\prime} a_{1}^{2}$. As we already mentioned in Remark 2.4, its knot Floer homology at the top filtration level is the same as the one of $\bar{a}_{2} P^{\prime} a_{1}$, to which we can apply the induction hypothesis.

If the last letter in $P$ is $a_{1}$, then $P$ can be written as $P^{\prime} a_{3} a_{1}$. We consider the skein relation for

$$
L_{-}=\bar{a}_{2} P^{\prime} a_{3} a_{1}, \quad L_{0}=\bar{a}_{2} P^{\prime} a_{3}, \quad L_{+}=\bar{a}_{2} P^{\prime} a_{3} \bar{a}_{1} .
$$

We have $\bar{a}_{2} P^{\prime} a_{3} \bar{a}_{1} \sim P^{\prime} a_{3} \bar{a}_{1} \bar{a}_{2}=P^{\prime} a_{3} \bar{\alpha}=P^{\prime} \bar{a}_{1}$. Length of $P^{\prime} \bar{a}_{1}$ is less than length of $\bar{a}_{2} P^{\prime} a_{3} a_{1}$, hence $\mathfrak{i}\left(L_{+}\right)<\mathfrak{i}\left(L_{-}\right)$. We have $\chi\left(L_{0}\right)=\chi\left(L_{-}\right)+1$. If the two strands in $L_{-}$belong to the same component, then $\left|L_{0}\right|=\left|L_{-}\right|+1$, and $\mathfrak{i}\left(L_{0}\right)=\mathfrak{i}\left(L_{-}\right)$; if the two strands in $L_{-}$belong to different components, then 
$\left|L_{0}\right|=\left|L_{-}\right|-1$, and $\mathfrak{i}\left(L_{0}\right)=\mathfrak{i}\left(L_{-}\right)-1$. In any case, we get an isomorphism between $\widehat{H F K}\left(L_{-}, \mathfrak{i}\left(L_{-}\right)\right)$and $\widehat{H F K}\left(L_{0}, \mathfrak{i}\left(L_{0}\right)\right)$. Now we apply the induction hypothesis to $L_{0}$.

Proposition 2.8 If $L$ is of the type $N P, N$ and $P$ are nonempty, then $\widehat{H F K}(L)$ is monic.

Proof We induct on $l(N)$. The case when $l(N)=1$ is the lemma above. Now we assume $l(N) \geq 2$, we can suppose the first letter in $N$ is $\bar{a}_{3}$, then $N=\bar{a}_{3} \bar{a}_{2} N^{\prime}$.

If the last letter in $P$ is $a_{1}, P=P^{\prime} a_{1}$. Then we consider the skein relation for

$$
L_{-}=a_{3} \bar{a}_{2} N^{\prime} P^{\prime} a_{1}, \quad L_{0}=\bar{a}_{2} N^{\prime} P^{\prime} a_{1}, \quad L_{+}=\bar{a}_{3} \bar{a}_{2} N^{\prime} P^{\prime} a_{1} .
$$

We have $a_{3} \bar{a}_{2} N^{\prime} P^{\prime} a_{1} \sim a_{1} a_{3} \bar{a}_{2} N^{\prime} P^{\prime}=a_{3} N^{\prime} P^{\prime}$. Same argument as before shows that $\widehat{H F K}\left(L_{0}, \mathfrak{i}\left(L_{0}\right)\right) \cong \widehat{H F K}\left(L_{+}, \mathfrak{i}\left(L_{+}\right)\right)$. We then apply the induction hypothesis to $L_{0}$.

If the last letter in $P$ is $a_{2}, P=P^{\prime} a_{2}$. Consider the skein relation for

$$
L_{-}=a_{3} \bar{a}_{2} N^{\prime} P^{\prime} a_{2}, \quad L_{0}=\bar{a}_{2} N^{\prime} P^{\prime} a_{2}, \quad L_{+}=\bar{a}_{3} \bar{a}_{2} N^{\prime} P^{\prime} a_{2} .
$$

$L_{0}$ can be reduced to $N^{\prime} P^{\prime}$, hence we get our conclusion as before, by applying the induction hypothesis to $L_{-}=\bar{a}_{2} N^{\prime} P^{\prime} a_{2} a_{3}$.

Proposition 2.9 If $L$ is in the form $P, P=\left(a_{1} a_{2} a_{3}\right)^{t}$ or $\left(a_{1} a_{2} a_{3}\right)^{t} a_{1}, t \geq 1$. Then

$$
\widehat{H F K}(L, \mathfrak{i}(L)) \cong \mathbb{Z} \oplus \mathbb{Z}
$$

Proof Suppose $P=\left(a_{1} a_{2} a_{3}\right)^{t}$, consider the skein relation for

$$
L_{-}=a_{2}\left(a_{1} a_{2} a_{3}\right)^{t}, \quad L_{0}=\left(a_{1} a_{2} a_{3}\right)^{t}, \quad L_{+}=\bar{a}_{2}\left(a_{1} a_{2} a_{3}\right)^{t} .
$$

$L_{-}$can be rewritten as $\alpha P^{\prime}$, which was considered in Proposition 2.6, and $L_{+}$ is of the type considered in Lemma 2.7. Then $\widehat{H F K}\left(L_{0}, \mathfrak{i}\left(L_{0}\right)\right)$ is fit into the exact triangle:

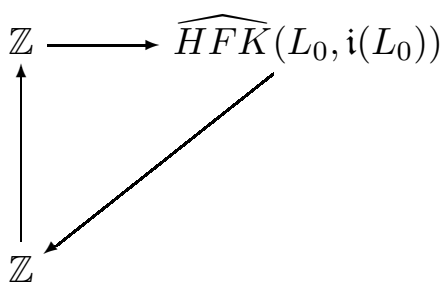


By [5], $\widehat{H F K}\left(L_{0}, \mathfrak{i}\left(L_{0}\right)\right) \otimes \mathbb{Q}$ is nontrivial. One then easily sees that

$$
\widehat{H F K}\left(L_{0}, \mathfrak{i}\left(L_{0}\right)\right) \cong \mathbb{Z} \oplus \mathbb{Z}
$$

The case when $P=\left(a_{1} a_{2} a_{3}\right)^{t} a_{1}$ can be reduced to the previous one by Remark 2.4 .

Proof of Theorem 2.5 Our theorem now follows from Remark 2.4, Proposition 2.6, Proposition 2.8 and Proposition 2.9.

Remark 2.10 With more care, one can get some information of the absolute grading. For example, in Proposition 2.6, the topmost term lies at grading level $l(P)+\frac{|L|-1}{2}$.

Remark 2.11 Our proof does not really need the fact that the Bennequin surface of Xu's word is the minimal Seifert surface. This fact can be proved inductively by our argument.

Remark 2.12 During the course of this work, we noted the paper [12], in which Stoimenow studied the skein polynomial of closed 3-braids, also using Xu's theorem. Our result here should be compared with Stoimenow's work.

\section{Proof of the main theorem}

Lemma 3.1 Suppose $w$ is a shortest word for $L, w$ is not necessarily in Xu's form. If the array $\bar{a}_{1} a_{3} a_{1} a_{2}$ appears in $w$, then we can replace the array by $\bar{a}_{1} a_{2}$, thus get a new word $w^{\prime}$, with closure $L^{\prime}$. Then $L$ is fibred with fiber $B_{w}$, if and only if $L^{\prime}$ is fibred with fiber $B_{w^{\prime}}$.

Proof We draw the local picture of the closed braid near the array $\bar{a}_{1} a_{3} a_{1} a_{2}$ as in Figure 2a. We leave the reader to figure out the local Bennequin surface. As in [4], we thicken $B_{w}$ to a sutured manifold $B_{w} \times I$, and consider its complementary sutured manifold. In Figure $2 \mathrm{~b}$, we draw the suture (as curves) on the boundary of the handlebody $B_{w} \times I$. There is an obvious product disk in the complementary sutured manifold, namely, the disk bounded by the dashdotted rectangle specified in Figure 2b. 


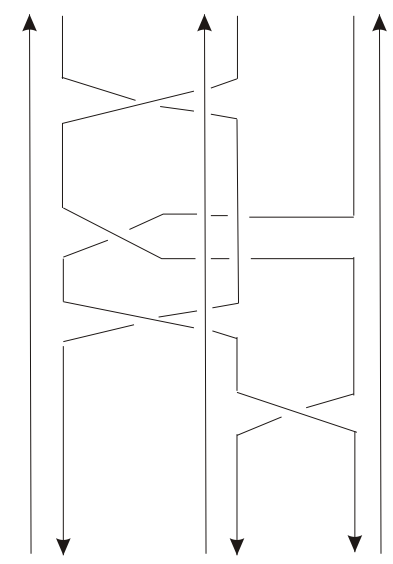

Figure 2a

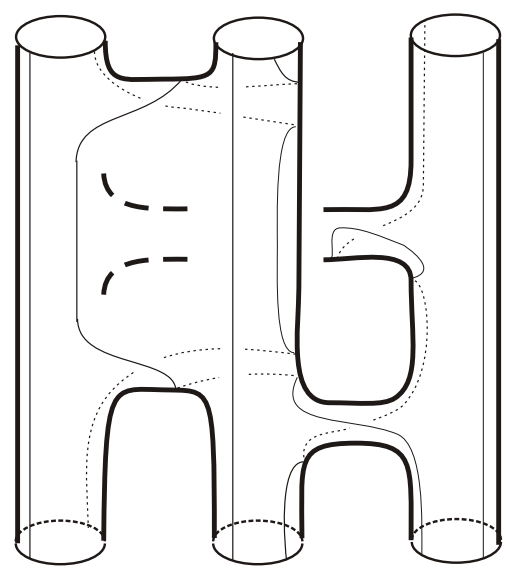

Figure 3a

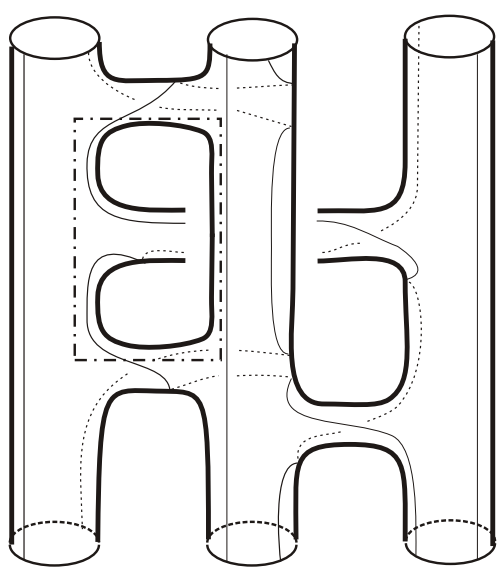

Figure $2 \mathrm{~b}$

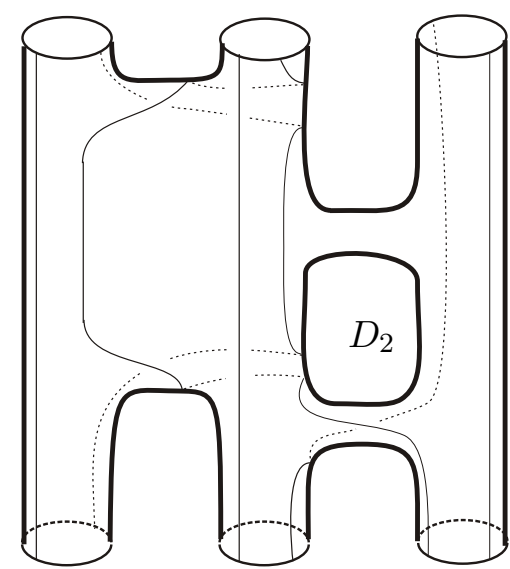

Figure $3 b$

We decompose the complementary sutured manifold along the product disk, thus get Figure 3a. After an isotopy, we get Figure 3b, where the product disk $D_{2}$ is clearer.

Now decompose the complementary sutured manifold in Figure 3b, thus get Figure 4a. After an isotopy, we get Figure $4 \mathrm{~b}$, which is just the local picture of a Bennequin surface near the array $\bar{a}_{1} a_{2}$.

Our conclusion holds by Lemma 2.2 in [4]. 


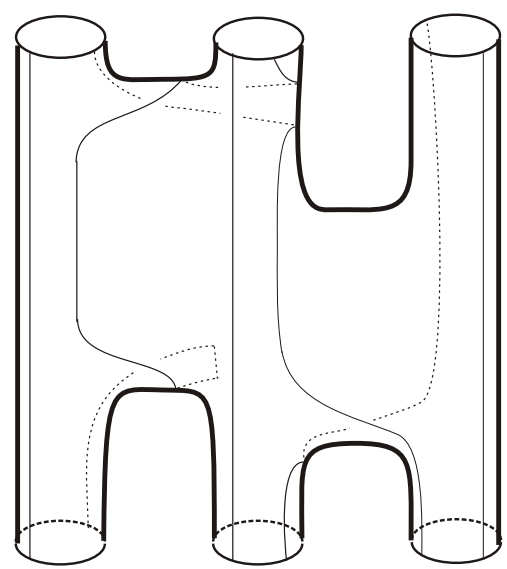

Figure $4 \mathrm{a}$

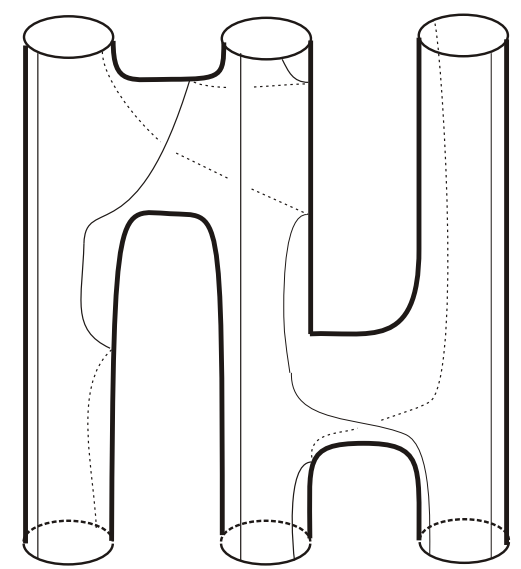

Figure $4 b$

Lemma 3.2 Suppose $w$ is a shortest word for $L, w$ is not necessarily in Xu's form. If the array $\bar{a}_{1} a_{2} a_{3} a_{1} a_{2}$ appears in $w$, then we can replace the array by $\bar{a}_{1} a_{2}$, thus get a new word $w^{\prime}$, with closure $L^{\prime}$. Then $L$ is fibred with fiber $B_{w}$, if and only if $L^{\prime}$ is fibred with fiber $B_{w^{\prime}}$.

Proof We note that the algebraic relation

$$
\cdots \bar{a}_{1} a_{2} \cdots=\cdots a_{3} \bar{a}_{1} \cdots
$$

also gives a local isotopy of the Bennequin surfaces. We have $\bar{a}_{1} a_{2} a_{3} a_{1} a_{2}=$ $a_{3} \bar{a}_{1} a_{3} a_{1} a_{2}$. By Lemma 3.1, we can replace $a_{3} \bar{a}_{1} a_{3} a_{1} a_{2}$ by $a_{3} \bar{a}_{1} a_{2}$. Now $a_{3} \bar{a}_{1} a_{2}=\bar{a}_{1} a_{2}^{2}$, we get our conclusion by Remark 2.4 .

Proposition 3.3 Suppose $w=N P$ is a shortest word in Xu's form for $L$. $l(N), l(P)>0$. Then $L$ is fibred with fiber $B_{w}$.

Proof Without loss of generality, can assume $\bar{N}, P$ are strictly increasing, and the last letter in $N$ is $\bar{a}_{1}$. By Lemma 3.1 and Lemma 3.2, we can replace $P$ by one of the following words: $a_{3}, a_{3} a_{1}, a_{2}, a_{2} a_{3}, a_{2} a_{3} a_{1}$. Then consider $\bar{P} \bar{N}$. By cyclically permuted versions of Lemma 3.1 and Lemma 3.2, we can replace $\bar{N}$ by a word with length $\leq 3$. Now there are only finitely many cases for $N P$ we need to consider. (We note that $N P$ should be cyclically reduced, this restriction also reduces the cases.) For these cases, we verify our theorem directly. 
Proof of Theorem 1.2 By Proposition 2.6, Proposition 3.3, we only need to consider the case that $w=P, P$ is strictly increasing. The case that $l(P) \leq 1$ is easy. If $l(P)=3 t+2,(t \geq 0$,$) then it is conjugated to the form in Proposition$ 2.6. If $l(P)=3 t+3$ or $l(P)=3 t+4(t \geq 0)$, then one can conjugate the word so that it is started with $a_{1}$ and ended with $a_{3}$. Now $a_{2} P$ is fibred by Proposition 2.6.

\section{References}

[1] J Birman, W Menasco, Studying links via closed braids. III. Classifying links which are closed 3-braids, Pacific J. Math. 161 (1993), no. 1, 25-113

[2] S Friedl, T Kim, Thurston norm, fibered manifolds and twisted Alexander polynomials, arXiv:math.GT/0505594

[3] D Gabai, The Murasugi sum is a natural geometric operation, Low-dimensional topology (San Francisco, Calif., 1981), 131-143, Contemp. Math., 20, Amer. Math. Soc., Providence, RI, 1983

[4] D Gabai, Detecting fibred links in $S^{3}$, Comment. Math. Helv. 61 (1986), no. 4, 519-555

[5] Y Ni, A note on knot Floer homology of links, arXiv:math.GT/0506208

[6] Y Ni, Sutured Heegaard diagram for knots, arXiv:math.GT/0507440

[7] P Ozsváth, Z Szabó, Holomorphic disks and knot invariants, Adv. Math. 186 (2004), no. 1, 58-116

[8] P Ozsváth, Z Szabó, Heegaard Floer homologies and contact structures, arXiv:math.SG/0210127

[9] P Ozsváth, Z Szabó, Holomorphic disks and genus bounds, Geom. Topol. 8 (2004), 311-334 (electronic)

[10] P Ozsváth, Z Szabó, Heegaard diagrams and holomorphic disks, Different faces of geometry, 301-348, Int. Math. Ser. (N. Y.), Kluwer/Plenum, New York, 2004

[11] J Rasmussen, Floer homology and knot complements, Harvard Thesis, arXiv:math.GT/0306378

[12] A Stoimenow, The skein polynomial of closed 3-braids, J. Reine Angew. Math. 564 (2003), 167-180

[13] A Stoimenow, Properties of closed 3-braids, preprint

[14] $\mathbf{P} \mathbf{X u}$, The genus of closed 3-braids, J. Knot Theory Ramifications 1 (1992), no. $3,303-326$

Department of Mathematics, Princeton University, Princeton, New Jersey 08544

Email: yni@math.princeton.edu 